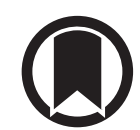

CrossMark

\title{
Acute exacerbation of idiopathic pulmonary fibrosis: international survey and call for harmonisation
}

\author{
Michael Kreuter ${ }^{1,2,36}$, Markus Polke ${ }^{1,36}$, Simon L.F. Walsh ${ }^{3}$, Johannes Krisam $^{4}$, \\ Harold R. Collard ${ }^{5}$, Nazia Chaudhuri ${ }^{6}$, Sergey Avdeev ${ }^{7}$, Jürgen Behr ${ }^{8,9}$, \\ Gregory Calligaro (10 ${ }^{10}$, Tamera Corte ${ }^{11}$, Kevin Flaherty ${ }^{12}$, \\ Manuela Funke-Chambour ${ }^{13}$, Martin Kolb (10 ${ }^{14}$, Yasuhiro Kondoh ${ }^{15}$, \\ Toby M. Maher ${ }^{16,17}$, Maria Molina Molina ${ }^{18,19}$, Antonio Morais (10) ${ }^{20}$, \\ Catharina C. Moor ${ }^{21}$, Julie Morisset ${ }^{22}$, Carlos Pereira ${ }^{23}$, Silvia Quadrelli2 ${ }^{24,25}$, \\ Moises Selman (1026, Argyrios Tzouvelekis ${ }^{27}$, Claudia Valenzuela ${ }^{28}$, \\ Carlo Vancheri ${ }^{29}$, Vanesa Vicens-Zygmunt ${ }^{30,31}$, Julia Wälscher ${ }^{1}$, Wim Wuyts (1032, \\ Marlies Wijsenbeek ${ }^{21,37}$, Vincent Cottin $\mathbb{1}^{33,34,37}$ and Elisabeth Bendstrup ${ }^{35,37}$
}

@ERSpublications

Lack of focussed international guidelines for management of acute exacerbation of IPF results in global variability in prevention, diagnosis and treatment strategies. Global trials are urgently needed to inform international specific guidelines for AE-IPF. http://bit.ly/3a8FB5i

Cite this article as: Kreuter M, Polke M, Walsh SLF, et al. Acute exacerbation of idiopathic pulmonary fibrosis: international survey and call for harmonisation. Eur Respir J 2020; 55: 1901760 [https://doi.org/ 10.1183/13993003.01760-2019].

ABSTRACT Acute exacerbation of idiopathic pulmonary fibrosis (AE-IPF) is an often deadly complication of IPF. No focussed international guidelines for the management of AE-IPF exist. The aim of this international survey was to assess the global variability in prevention, diagnostic and treatment strategies for AE-IPF.

Pulmonologists with ILD expertise were invited to participate in a survey designed by an international expert panel.

509 pulmonologists from 66 countries responded. Significant geographical variability in approaches to manage AE-IPF was found. Common preventive measures included antifibrotic drugs and vaccination. Diagnostic differences were most pronounced regarding use of Krebs von den Lungen- 6 and viral testing, while high-resolution computed tomography, brain natriuretic peptide and D-dimer are generally applied. High-dose steroids are widely administered (94\%); the use of other immunosuppressant and treatment strategies is highly variable. Very few (4\%) responders never use immunosuppression. Antifibrotic treatments are initiated during AE-IPF by 67\%. Invasive ventilation or extracorporeal membrane oxygenation are mainly used as a bridge to transplantation. Most physicians educate patients comprehensively on the severity of AE-IPF (82\%) and consider palliative care (64\%).

Approaches to the prevention, diagnosis and treatment of AE-IPF vary worldwide. Global trials and guidelines to improve the prognosis of AE-IPF are needed. 


\section{Introduction}

Idiopathic pulmonary fibrosis (IPF) is a chronic and progressive fibrosing interstitial lung disease with a $20-40 \%$ five-year survival rate and a median survival time of 2-5 years [1]. Acute exacerbation of IPF (AE-IPF) is often the primary cause of death in patients with this disorder [2].

AE-IPF is defined as an acute, clinically significant respiratory deterioration characterised by evidence of new widespread alveolar abnormality. Diagnostic criteria are previous or concurrent diagnosis of IPF, acute worsening or development of dyspnoea within 1 month duration, computed tomography with new bilateral ground-glass opacity and/or consolidation on a background pattern with usual interstitial pneumonia pattern and deterioration not fully explained by cardiac failure or fluid overload [3]. The incidence varies between 7 and 32\%, and current evidence suggests that up to $46 \%$ of deaths in IPF are associated with AE-IPF [6]. In-hospital mortality after AE-IPF exceeds 50\% [2, 4, 5], and the median survival after AE-IPF is approximately 3 to 4 months [6]. AE-IPF may be either triggered, e.g. by infection, post-procedural/post-operative, drug toxicity, aspiration or might be idiopathic [6]. Currently, no focussed international guidelines exist regarding the prevention, diagnosis or therapy of AE-IPF [3,6]. While the clinical practice guideline for IPF provides a weak recommendation for treatment with steroids, this recommendation is based on expert opinion and there is no specific guidance on dose, route and duration or diagnostic or therapeutic approaches. Data from clinical trials especially on the treatment of AE-IPF are sparse and, currently, there are no large randomised controlled trial data on AE-IPF available.

We hypothesised that clinical approaches to the investigation and management of suspected AE-IPF might vary substantially, which may inform us about priority research questions to be addressed. Therefore, this study aimed to explore preventive, diagnostic and therapeutic strategies towards AE-IPF in an international group of respiratory physicians to guide future clinical trial design and recommendations for this condition.

\section{Materials and methods}

Questionnaire and participating physicians

To identify the items to be included in this survey, we conducted literature research on diagnostics, therapy, prevention and management of AE-IPF on www.ncbi.nlm.nih.gov/pubmed, https://scholar.google.com and

Affiliations: ${ }^{1}$ Center for Interstitial and Rare Lung Diseases, Pneumology, Thoraxklinik, University of Heidelberg, Heidelberg, Germany. ${ }^{2}$ Member of the German Center for Lung Research (DZL), Germany. ${ }^{3}$ National Heart and Lung Institute, Imperial College, London, UK. ${ }^{4}$ Institute of Medical Biometry and Informatics, University of Heidelberg, Heidelberg, Germany. ${ }^{5}$ Dept of Medicine, University of California San Francisco, San Francisco, CA, USA. ${ }^{6}$ North West Interstitial Lung Disease Unit, Manchester University NHS Foundation Trust, Manchester, UK. ${ }^{7}$ Sechenov First Moscow State Medical University, Moscow, Russia. ${ }^{8}$ Dept of Internal Medicine V, Ludwig-Maximilians University of Munich, Munich, Germany. ${ }^{9}$ Asklepios Clinic Gauting, Member of the German Center for Lung Research, Gauting, Germany. ${ }^{10}$ Division of Pulmonology, Dept of Medicine, University of Cape Town, Cape Town, South Africa. ${ }^{1}$ Royal Prince Alfred Hospital, University of Sydney, Sydney, Australia. ${ }^{12}$ Dept of Medicine, University of Michigan, Ann Arbor, MI, USA. ${ }^{13}$ Dept of Pulmonary Medicine, Bern University Hospital, University of Bern, Bern, Switzerland. ${ }^{14}$ Firestone Institute for Respiratory Health, Research Institute at St Joseph's Healthcare, Dept of Medicine, McMaster University, Hamilton, ON, Canada. ${ }^{15}$ Dept of Respiratory Medicine and Allergy, Tosei General Hospital, Seto, Japan.

${ }^{16}$ National Heart and Lung Institute, Imperial College London, UK. ${ }^{17}$ Interstitial Lung Disease Unit, Royal Brompton and Harefield NHS Foundation Trust, London, UK. ${ }^{18}$ Institut d'Investigació Biomèdica de Bellvitge (IDIBELL), University Hospital of Bellvitge, L'Hospitalet de Llobregat, Barcelona, Spain. ${ }^{19}$ Centro de Investigación Biomédica en Red Enfermedades Respiratorias (CIBERES), Madrid, Spain. ${ }^{20}$ Pneumology Dept, Centro Hospitalar São João, Faculdade de Medicina, Universidade do Porto, Porto, Portugal. ${ }^{21}$ Centre for Interstitial Lung Diseases and Sarcoidosis, Dept of Respiratory Medicine, Erasmus University Medical Center, Rotterdam, The Netherlands. ${ }^{22}$ Département de Médecine, Centre Hospitalier de l'Université de Montréal, Montréal, QC, Canada. ${ }^{23}$ Lung Disease Dept, Paulista School of Medicine, Federal University of São Paulo, São Paulo, Brazil. ${ }^{24}$ Hospital Británico, Buenos Aires, Argentina. ${ }^{25}$ Sanatorio Güemes, Buenos Aires, Argentina. ${ }^{26}$ Instituto Nacional de Enfermedades Respiratorias Ismael Cosío Villegas, Tlalpan, Mexico City, Mexico.

${ }^{27}$ First Academic Respiratory Dept, Sotiria General Hospital for Thoracic Diseases, University of Athens, Athens, Greece. ${ }^{28}$ Instituto de Investigación Princesa, Hospital Universitario de La Princesa, Madrid, Spain. ${ }^{29}$ Regional Referral Centre for Rare Lung Diseases, A.O.U. Policlinico-Vittorio Emanuele, University of Catania, Catania, Italy. ${ }^{30}$ Pneumology Research Group, IDIBELL, L'Hospitalet de Llobregat, Barcelona, Spain. ${ }^{31}$ Unit of Interstitial Lung Diseases, Department of Pneumology, University Hospital of Bellvitge, L'Hospitalet de Llobregat, Barcelona, Spain. ${ }^{32}$ Unit for Interstitial Lung Diseases, Dept of Respiratory Diseases, University Hospitals Leuven, Leuven, Belgium. ${ }^{33}$ National Coordinating Reference Center for Rare Pulmonary Diseases, Louis Pradel Hospital, Lyon, France. ${ }^{34}$ Hospices Civils de Lyon, UMR754, University Claude Bernard Lyon 1, Lyon, France. ${ }^{35}$ Dept of Respiratory Diseases and Allergy, Aarhus University Hospital, Aarhus C, Denmark. ${ }^{36}$ Equal contribution. ${ }^{37}$ Shared senior authorship.

Correspondence: Michael Kreuter, Center for Interstitial and Rare Lung Diseases, Thoraxklinik, University of Heidelberg, Röntgenstraße 1, 69126 Heidelberg, Germany. E-mail: kreuterवuni-heidelberg.de 
others (supplementary file 1). Next, an expert panel was created, comprising respiratory physicians with expertise in the diagnosis and management of ILD working in specialist ILD centres and a track record of publication in this field, to participate in an email-based interview to structure the survey. The final questionnaire consisted of 20 questions regarding diagnosis, treatment and prevention of AE-IPF and suggested future perspectives in AE-IPF research (supplementary file 2). Additionally, optional questions were included on working place (including ILD-expert centres versus non-expert centres), country of origin, number of patients with IPF under care and estimated number of AE-IPF seen.

An internet search was performed from July 1, 2017 to November 30, 2017 to identify practising respiratory physicians worldwide with interest in ILD. This search included the European Respiratory Society assembly on Diffuse Parenchymal Lung Disease, the American Thoracic Society assembly on Clinical Problems, the Japanese Respiratory Society assembly on Diffuse Parenchymal Lung Disease and participants of the IPF Project Consortium (www.theipfproject.com) [7]. Nationality, academic status (working at a university hospital or not) or subspecialist interests within respiratory medicine did not influence inclusion eligibility. Pulmonologists were invited to participate via an e-mail link. The questionnaire was available on the online survey tool SurveyMonkey from December 2017 to April 2018.

\section{Statistical analysis}

For questions with categorical answers, absolute and relative frequencies were calculated and differences between continents were assessed using Chi-squared tests. For questions with answers on a continuous scale, median, first and third quartile, minimum and maximum were determined and differences between continents were assessed using Kruskal-Wallis tests. Due to the exploratory nature of this survey, all resulting $\mathrm{p}$-values are solely to be interpreted descriptively and no adjustment for multiple testing was conducted. A p-value $<0.05$ was regarded as statistically significant. All analyses were conducted using $\mathrm{R}$ v.3.4.2 (http://r-project.org).

\section{Results}

\section{Participants}

Overall, 509 pulmonologists from 66 countries responded. 42.6\% $(\mathrm{n}=217)$ were from Europe, 26.7\% $(\mathrm{n}=136)$ from Asia, 11.2\% $(\mathrm{n}=57)$ from North America, 9.8\% $(\mathrm{n}=50)$ from South America, 4.9\% $(\mathrm{n}=25)$ from Australia (including New Zealand), 1\% $(n=5)$ from Africa and 3.7\% $(n=19)$ remained anonymous (figure 1a and b). 66\% of the participants worked in a specialised ILD centre/university hospital, $28 \%$ in general pulmonology departments/non-university centres and $1 \%$ on an intensive care unit $(5.3 \%$ in others). The average number of IPF patients under care was 130; the estimated median number of patients with AE-IPF seen per year was 18 . Overall, 1-year mortality of patients with AE-IPF was estimated to be $50-80 \%$ by $41.9 \%, 20-50 \%$ by $35.1 \%,>80 \%$ by $14.7 \%$ and $<20 \%$ by $8.4 \%$.

\section{Diagnostic procedures for AE-IPF}

High-resolution computed tomography (HRCT) (multi-slice thin-section CT, without contrast media) was performed by $76 \%$ participants with the highest rates in Asia (91\%) and lowest in Europe (67\%). CT with contrast media was applied less frequently (34\%) but even in the absence of a clinical suspicion of pulmonary embolism. Most physicians used it in Europe (45\%), fewest in Asia (20\%) and Africa (20\%).
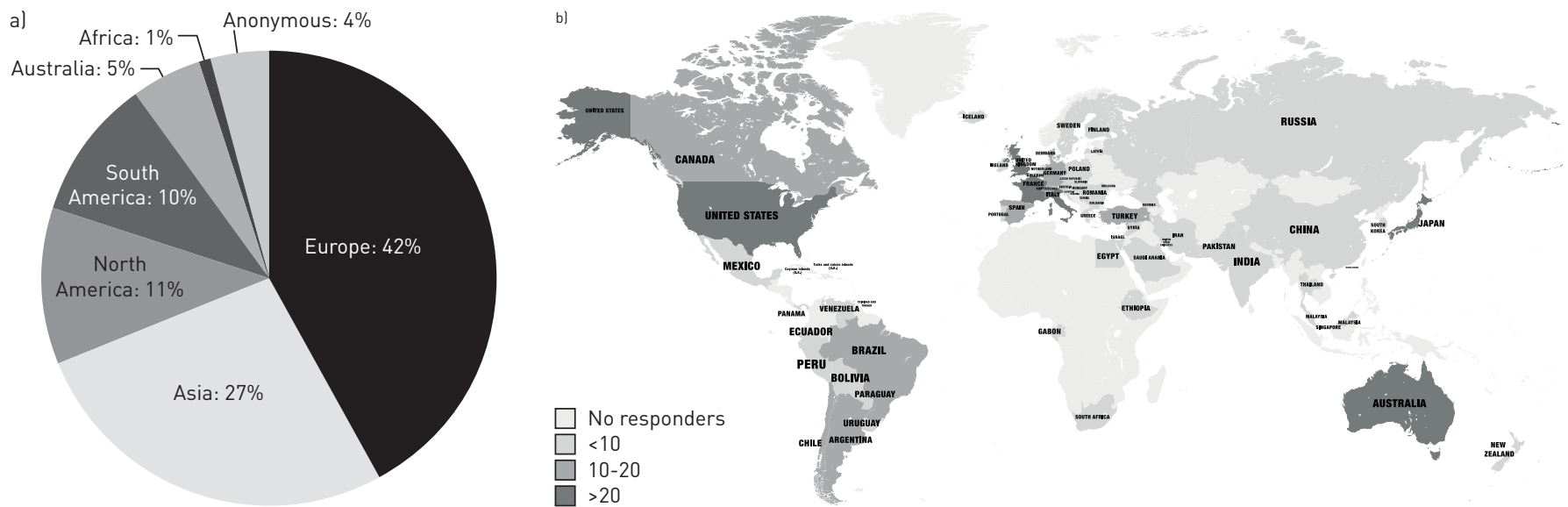

FIGURE 1 a) Participants ( $n=217(42 \%)$ from Europe, $n=136(27 \%)$ from Asia, $n=57$ (11\%) from North America, $n=50$ (10\%) from South America, $n=25(5 \%)$ from Australia, $n=5(1 \%)$ from Africa and $n=19(4 \%)$ remained anonymous). 
Echocardiography to screen for cardiac reasons for deterioration was used by $66 \%$. N-terminal pro-hormone of BNP (NT-proBNP)/BNP (72\%), D-dimer (64\%) and troponins (50\%) were used widely during the diagnostic workup of an AE-IPF. As a biomarker for AE-IPF, KL-6 was used in Asia (54\%), but not elsewhere.

Bronchoalveolar lavage (BAL) in the context of AE-IPF was always performed by $5.8 \%$, while the majority (70.5\%) only performed BAL in case of suspected infection. For microbiology assessments, mainly sputum was collected (85\%) while induced sputum was sampled by $14 \%$. Specific pathogen screening for influenza viruses (75.7\%), atypical bacterial pathogens (61.8\%) and Pneumocystis jirovecii (58.6\%) was common. Only a minority screened for other pathogens like respiratory syncytial virus (44.4\%), cytomegalovirus (37.8\%), Aspergillus spp. (37.6\%), Candida spp. (17\%) and tuberculosis (10.9\%). A minority (9.2\%) did not screen for any specific infections.

The main diagnostic procedures applied for AE-IPF, which vary significantly between the continents, are shown in figure 2 (further results can be seen in supplementary file 3, table S1).

\section{Treatment approaches for AE-IPF}

The majority of participating pulmonologists treated AE-IPF with methylprednisolone or equivalent with a dosage of 500-1000 mg per day for 3 days followed by a slow tapering (63\%), while $11 \%$ applied pulsed high-dose steroids for 3 days only. $31 \%$ used prednisolone with a dosage of $1 \mathrm{mg} \cdot \mathrm{kg}^{-1}$ per day followed by a slow tapering. On average, physicians treated AE-IPF with corticosteroids for 13 weeks.

Other immunosuppressive therapies were rarely used: 19\% use cyclophosphamide (intravenous bolus), $9 \%$ cyclosporine, $5 \%$ tacrolimus and $4 \%$ rituximab. Differences between continents in the use of immunomodulators were significant (supplementary file 3, table S2). For instance, cyclophosphamide was used by $28 \%$ in Asia and never in North America. Only a minority never treated AE-IPF with any immunosuppressive therapy (4\%).

Other therapies such as polymyxin B haemoperfusion, recombinant thrombomodulin and plasmapheresis/ plasma exchange were used primarily in Asia (supplementary file 3, table S2).

Antimicrobial therapy was commenced regularly by $56 \%$ with broad-spectrum antibiotics combined with macrolides. $23 \%$ only used antibiotic treatment in case of a clinical and/or laboratory indication of a bacterial infection.

In AE-IPF patients without previous antifibrotic therapy, most participants would have initiated such therapy (nintedanib: $21 \%$; pirfenidone: $14 \%$; either nintedanib or pirfenidone: $32 \%$ ), while $33 \%$ did not see an indication for an antifibrotic treatment in the acute setting. Most physicians (71\%) would have waited until clinical stabilisation before initiating antifibrotic therapy. In patients already on antifibrotic therapy at the time of AE-IPF, 76\% of respondents recommended its continuation, while a minority would have advised differently ( $4 \%$ discontinue, $3 \%$ reduce dose, $10 \%$ switch the antifibrotic drug). For gastro-oesophageal reflux disease (GORD), 19\% always initiated or increased antacid therapy during AE-IPF (supplementary file 3, table S2). The main management approaches are shown in figure 3.

In case of respiratory failure, invasive ventilation was offered to all patients by $9 \%$, and by $45 \%$ only to patients suitable for lung transplantation (LTX), as a bridge to LTX or in very selected other cases. Extracorporeal membrane oxygenation (ECMO) was offered to patients suitable for LTX as a bridge to LTX by $44 \%$, mostly in Europe (57\%) and fewest in Oceania (24\%). Critically ill patients with AE-IPF were offered high-flow oxygen by $81 \%$ and non-invasive ventilation (NIV) by $74 \%$. Palliative care was considered by $65 \%$. Differences in these approaches were again significant between continents (supplementary file 3, table S3).

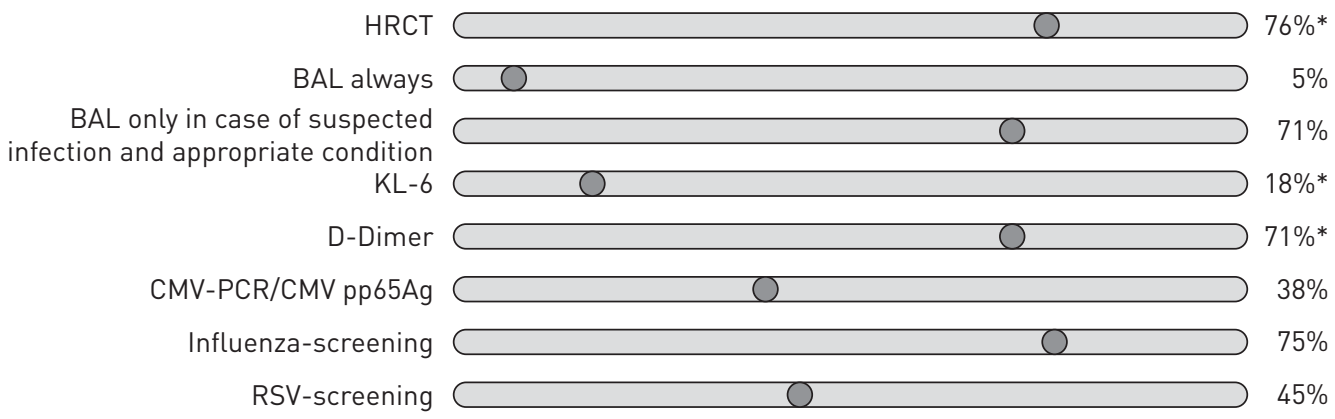

FIGURE 2 Main diagnostic procedures. *: $p \leqslant 0.0001$. 


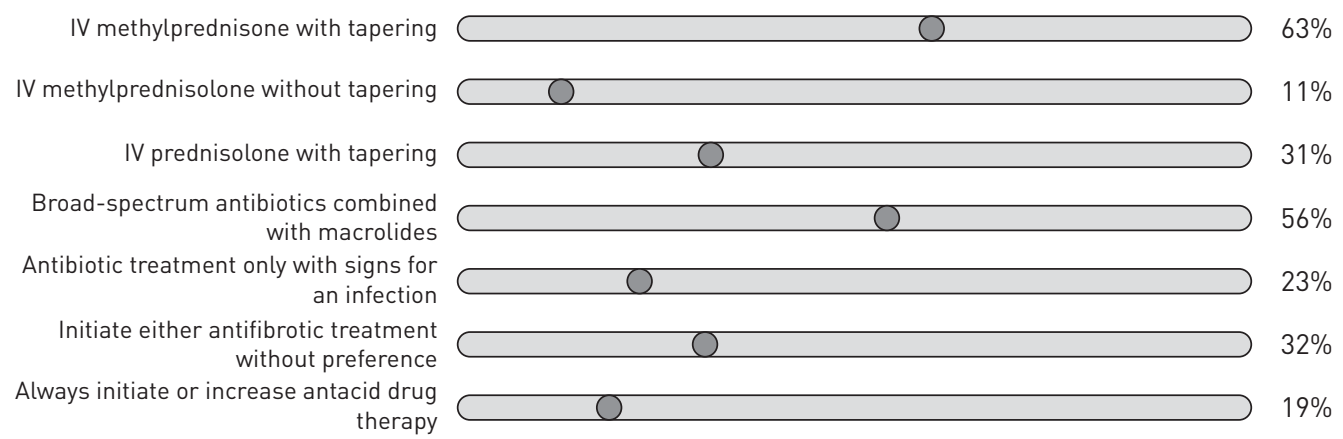

FIGURE 3 Main drug management approaches worldwide.

\section{Preventive strategies for AE-IPF}

Measures aiming to prevent AE-IPF were mainly vaccinations (i.e. influenza, pneumococcal) (93\%), antifibrotic therapy $(86 \%)$ and pulmonary rehabilitation or other forms of structured exercise therapy (58\%). Antacid drugs were prescribed by $52 \%$ respondents in all IPF patients. Only a minority used long-term azithromycin $(7 \%)$ or low-dose steroids $(\leqslant 10 \mathrm{mg})(4 \%)$. There were significant differences concerning prevention of AE-IPF between the continents (supplementary file 3, table S4). For instance, most physicians in Europe valued antifibrotic therapy as a preventive strategy (90\%), opposed to significantly fewer in Asia (79\%). Anticoagulation was only used by a minority (2\%).

In terms of planned surgical procedures, 69\% favoured preventive anaesthetic measures such as low tidal volume and avoidance of hyperoxygenation as well as regional anaesthesia over general anaesthesia when possible. $15 \%$ avoided any elective thoracic surgery. Differences between continents were again significant (figure 4 and supplementary file 3, table S4).

\section{Unmet needs in AE-IPF}

According to respondents, more research into treatment $(86 \%)$ and improving our understanding of the pathophysiology of AE-IPF (83\%) is needed. Furthermore, most respondents highlighted the need for consensus guideline recommendations for AE-IPF (79\%) and improved education and training of physicians (66.5\%) and patients and caregivers (60\%). 60\% see a need for improvement in the collaboration between different ILD specialists in general and 58\% in multidisciplinary strategies for diagnosing and discussion.

\section{Discussion}

Despite AE-IPF being a primary driver of mortality in IPF [3], evidence on prevention, additional diagnostic approaches besides HRCT and especially on treatment of this complication is sparse and evidence-based guidance particularly is missing. Our results, which are drawn from a large international group of respiratory physicians with expertise in the management of IPF, reveal many similarities, e.g. the use of HRCT for the diagnosis or the use of steroids for the treatment. But there are also significant differences in the approach to AE-IPF such as in the therapy strategies beyond steroids.

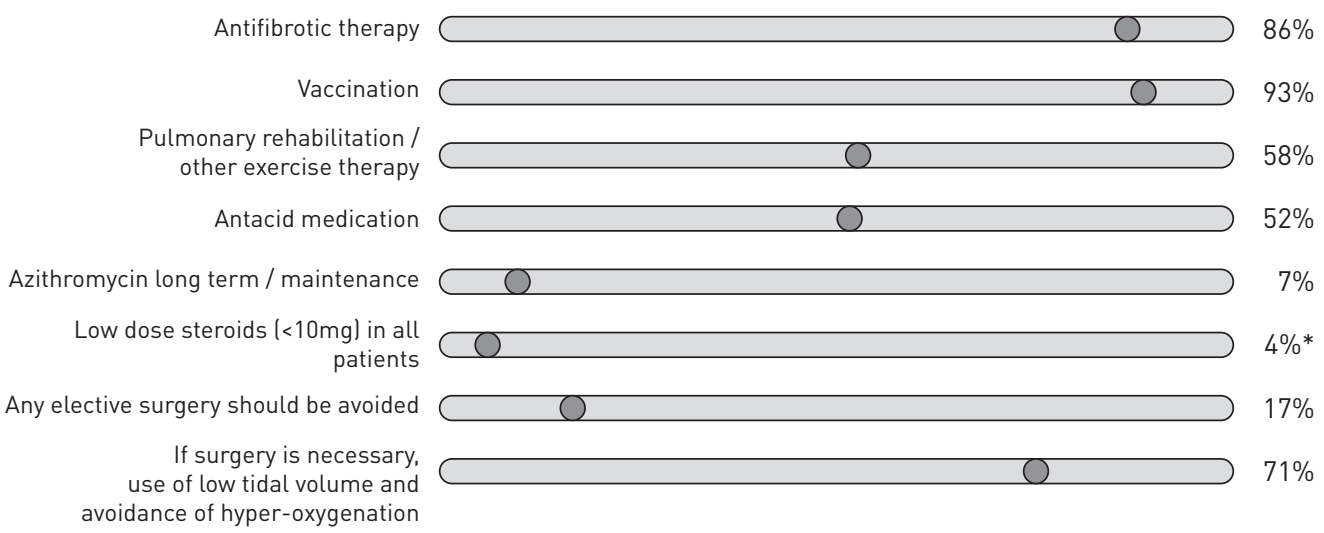

FIGURE 4 Preventive strategies. *: $p \leqslant 0.0001$. 
The majority of physicians use sputum analysis, HRCT, BNP and BAL in suspected infection and D-dimer for the differential diagnosis of AE-IPF, while diagnostic approaches differ regarding the use of KL-6 and viral testing.

As for treatment, high-dose steroids are widely administered, but the use of immunosuppressants and other strategies are highly variable. Very few respondents never use immunosuppression. There are also differences in the use of antifibrotic drugs in the context of AE-IPF. These results reflect an unmet need for clinical practice guidelines in this disorder.

Regarding diagnostic procedures in AE-IPF, surprisingly less than $80 \%$ of participants use HRCT despite the current definition of AE-IPF requiring evidence of new parenchymal changes on HRCT [3]. Moreover, HRCT might be critical in determining the prognosis as the extent and distribution of HRCT patterns during AE-IPF may predict outcome [8]. CT with contrast media is used by $34 \%$ of the participating physicians. Usually it is used in the process of excluding pulmonary embolism [9]. This is a very important tool because IPF patients are more likely to have a prothrombotic state compared with healthy individuals and this has an impact on survival [10].

Blood-based biomarkers in AE-IPF may also have prognostic value; KL-6 and serum decorin are reported to be predictive of AE-IPF in a Japanese population $[11,12]$. Based on low level evidence there are data proving that the bacterial load and the bacterial spectrum in patients with AE-IPF differs significantly from a stable disease [13], many clinicians search for pathogens; however, significant differences in treatment practice of viral and bacterial infections exist. A recent retrospective analysis of azithromycin was associated with a reduced mortality in AE-IPF compared with fluoroquinolones [14] but it remains unclear if the reduced mortality is explained by a possible harmful effect of fluoroquinolones. Furthermore, it is unclear if azithromycin may be useful in all forms of AE-IPF or only in AE-IPF caused by infection. Ding et al. [15] could show that the use of Procalcitonine may prevent an unnecessary use of antibiotics in AE-IPF.

Viruses are established triggers for acute respiratory failure in chronic diseases [16]; however, data on associations of viral infections in AE-IPF are contradictory [17, 18]. This may explain the rare use of antivirals such as aciclovir (1\%) and ganciclovir (2\%) in the treatment of AE-IPF.

The need for a general worldwide approach to treatment is mirrored in the lack of general guidance except an expert weak recommendation for treatment with steroids in the current international guideline [6]. In particular, more evidence for the use of high-dose steroids, commonly used in AE-IPF by the participants in this study, is required. While no data exist on outcomes associated with the use of steroids in AE-IPF, high-dose long-term steroid use was associated with an increased mortality in the PANTHER (Prednisone, Azathioprine, and N-Acetylcysteine for Pulmonary Fibrosis) trial [19] and a history of previous immunosuppression before IPF-AE has a negative impact on mortality [20]. Notably, some physicians use an even more potent anti-inflammatory treatment approach, e.g. cyclosporine A, intravenous cyclophosphamide and tacrolimus (mainly in Asia, rituximab mostly in North and South America), although there is low or very low evidence for the use of these treatments [21-25].; therefore, further trials are needed.

A majority of participants report prescription of antifibrotics as a way to prevent acute exacerbations. Controlled trials suggest that nintedanib may prolong the time to the first AE-IPF [26], while post hoc data on pirfenidone suggest that it may reduce the risk for respiratory-related hospitalisation [5]. There are no robust data whether antifibrotics ameliorate the course of AE-IPF in patients with acute respiratory failure. Current registries have to be analysed to obtain more information on this topic and the survival during and after AE-IPF; ILD experts already aim to do so [27].

Some prospective randomised trials are currently ongoing, such as a French study assessing the role of cyclophosphamide on top of pulsed steroids (NCT02460588), two studies assessing the effect of therapeutic plasma exchange, rituximab and intravenous immunoglobulins for severe AE-IPF patients admitted to the intensive care unit (ICU) (NCT03584802) and a study from Japan assessing the effect of recombinant thrombomodulin in addition to standard of care with steroid therapy (NCT02739165). These studies and others will hopefully address some of the key unresolved issues regarding treatment of AE-IPF.

The definition of idiopathic AE-IPF relies on the exclusion of other aetiologies, including infection [3]. However, only a minority used bronchoscopy with BAL. A recent study does not support this approach as a positive bronchoscopy only affected management in $13 \%$ of patients and resulted in a change of treatment in less than 5\%. In the same study, bronchoscopy resulted in a significant number of patients transferred to the ICU intubated and similarly a significant number of patients could not be extubated after the procedure [28]. In contrast, another report demonstrated the feasibility and safety of BAL aided by noninvasive ventilation (NIV) as a useful tool for differentiating or confirming triggered acute exacerbations [29]. It has to be discussed whether collection of bronchial secretion via bronchoscopy 
might be better tolerable and at least equally effective in suspected infection in AE-IPF; yet, this has to be evaluated in future trials.

The mortality of patients with AE-IPF admitted to the ICU, particularly in ventilated patients, is high [30]. Therefore, the international guidelines recommend avoiding the ICU in patients with AE-IPF (weak recommendation) [31]. NIV and high-flow oxygen are often initiated in critical ill patients but data on this are limited $[9,32]$. Other advanced therapies, such as invasive ventilation and ECMO, are usually only used as a bridge to LTX. This is in line with the current literature $[33,34]$ and thus included in the recommendations of the international guidelines [6].

Vaccinations theoretically play an important role in the prevention of AE-IPF but, while their use is recommended by the international guideline, there is a paucity of evidence to support this recommendation [35]. Also, it is not clear how local public health systems are dealing with these vaccinations and to what extent they are available.

Many physicians use antacid drugs as a preventive strategy for AE-IPF, although evidence on the role of antacids in IPF is controversial. LEE et al. [36] reported a higher pepsin level in the BAL of patients with AE-IPF compared to patients with stable diseases and also showed a positive impact of antacid drugs on the course of IPF in retrospective analyses $[37,38]$. However, recent studies could not support this effect and reported potentially higher rates of respiratory infections [39] and AE-IPF [40]. Only a few physicians use low-dose steroids as a preventive strategy for AE-IPF. This is in line with the international guideline that does not recommend the use of steroids beyond AE-IPF [31]. Amongst other data, this recommendation is based on the results of the PANTHER trial that demonstrated an increased risk of hospitalisation and death for patients receiving combination therapy with $N$-acetylcyteine, azathioprine and prendnisolone compared with controls [19]. Moreover, the use of corticosteroids does not have a positive effect on the outcome of IPF patients who receive nintedanib [41]. In the end, there are no data proving a benefit for the indication for steroids in the prevention of AE-IPF.

Even though IPF patients are more likely to have a prothrombotic state (as mentioned previously) [10] and the coagulation cascade was recognised as an initiator of fibrosis, there are data showing that it seems comprehensible that nearly no one uses anticoagulation for prevention of an exacerbation. Noth et al. [42] showed that the use of vitamin $\mathrm{K}$ antagonist warfarin in IPF patients lead to a decline in survival. This was also shown in patients who received oral anticoagulation, mainly vitamin $\mathrm{K}$ antagonists, for other medical reasons [43].

Most of the respondents identify the unmet needs of AE-IPF in the survey. Not only are treatment trials urgently needed but also trials addressing the pathophysiology of AE-IPF have to be expanded and an improved communication and collaboration between ILD specialists has to be supported.

Our survey has several limitations. Although there was a significant contribution of pulmonologists from all parts of the world, it is based on a survey of physicians and not on objective evaluation of management and practices. Participation took place on voluntary basis and may not reflect the general practice in the respective countries/continents. While there was a significant contribution of pulmonologists from most parts of the world, there were only a few participants from Africa. Also, it has to be mentioned that certain variability in the approach of AE-IPF has to be associated with different local possibilities: between sites but also continents/countries. Especially, access to treatments, such as immunomodulation like cyclophosphamide, cyclosporine or tacrolimus, antifibrotic drugs or ECMO might be limited in some countries.

Furthermore, this study aimed to survey international habits on diagnosis and treatment of AE-IPF, it was unable to assess reliable information on incidences and outcomes of AE-IPF in the respective countries. This should be addressed in future work analysing current registries.

Not all aspects of the management of AE-IPF could be addressed in the questionnaire. Our report also has strengths as we managed to get responses from all continents and from a significant number of physicians. The questionnaire was anonymous and therefore answers are anticipated to be less biased.

In conclusion, the heterogeneity of management of AE-IPF as found in this international survey reflects the lack of evidence and focussed guidelines on important aspects of the management of AE-IPF. This strongly calls for research, education and collaborations between ILD specialists around the world to find new ways to approach this deadly complication of IPF.

Acknowledgements: We would like to thank all participants who generously agreed to participate in this study.

Conflict of interest: M. Kreuter reports grants and personal fees from Roche, Galapagos and Boehringer, outside the submitted work. M. Polke has nothing to disclose. S.L.F. Walsh reports personal fees for consultancy from 
Sanofi-Aventis, Galapagos and OSIC, personal fees for advisory board work from Roche, grants and personal fees for steering committee work from Boehringer Ingelheim, personal fees for lectures from Bracco, outside the submitted work. J. Krisam has nothing to disclose. H.R. Collard reports personal fees from Bayer, Boehringer Ingelheim, Bristol-Myers Squibb, Global Blood Therapeutics, ImmuneWorks, Navitor, Parexel, Prometic, Toray, Unity, Patara, Veracyte, Roche/Genentech, aTyr, Advance Medical and MedImmune, grants from Pulmonary Fibrosis Foundation, grants and personal fees from Three Lakes Partners, outside the submitted work. N. Chaudhuri reports grants from Boehringer Ingelheim and Roche, educational support from Boehringer Ingelheim and Roche, outside the submitted work. S. Avdeev has nothing to disclose. J. Behr has nothing to disclose. G. Calligaro has nothing to disclose. T. Corte reports grants, personal fees for lectures and advisory board work, and travel support from Boehringer, grants and personal fees for lectures and advisory board work from Roche, grants from Galapagos, Actelion, Bayer and Sanofi, personal fees for advisory board work from AstraZeneca, outside the submitted work. K. Flaherty reports grants and personal fees from Boehringer Ingelheim and Roche/Genentech, personal fees from Bellerophon, Respivant, Veracyte, Sanofi-Genzyme, Blade Therapeutics and Celgene, outside the submitted work. M Funke-Chambour has nothing to disclose. M. Kolb reports grants and personal fees for consultancy and lectures from Roche and Boehringer Ingelheim, grants and personal fees for consultancy from GSK, Gilead and Prometic, grants from Actelion, Respivert, Alkermes and Pharmaxis, personal fees for consultancy from Genoa, Indalo and Third Pole, outside the submitted work. Y. Kondoh reports advisory board fees and lecture fees from Asahi Kasei Pharma Corp. and Boehringer Ingelheim Co., Ltd, advisory board fees from Janssen Pharmaceutical K.K., lecture fees from Eisai Inc., Kyorin Pharmaceutical Co., Ltd, Mitsubishi Tanabe Pharma, Novartis Pharma K.K. and Shionogi and Co., Ltd, outside the present work. T.M. Maher has, via his institution, received industry-academic funding from GlaxoSmithKline R\&D and UCB, and has received consultancy or speaker fees from Apellis, AstraZeneca, Bayer, Blade Therapeutics, Boehringer Ingelheim, Bristol-Myers Squibb, Galapagos, GlaxoSmithKline R\& D, Indalo, Novartis, Pliant, ProMetic, Respivnat, Roche, Samumed and UCB. M. Molina Molina reports grants and personal fees from Roche, Boehringer Ingelheim and Esteve-Teijin, grants from GSK and AstraZeneca, personal fees from Pfizer and Chiesi, outside the submitted work. A. Morais has nothing to disclose. C.C. Moor has nothing to disclose. J. Morisset reports personal fees from Hoffmann La Roche and Boehringer Ingelheim, outside the submitted work. C. Pereira has nothing to disclose. S. Quadrelli has nothing to disclose. M. Selman has nothing to disclose. A. Tzouvelekis has nothing to disclose. C. Valenzuela reports personal fees for advisory board work and lectures from Roche and Boehringer Ingelheim, personal fees for advisory board work from Galapagos, outside the submitted work. C. Vancheri reports grants and personal fees from Roche and Boehringer Ingelheim, outside the submitted work. V. Vicens-Zygmunt has nothing to disclose. J. Wälscher has nothing to disclose. W. Wuyts has nothing to disclose. M. Wijsenbeek reports grants and fees paid to institution from Boehringer Ingelheim and Hoffman la Roche, fees paid to institution from Galapagos and Novartis, outside the submitted work. V. Cottin reports personal fees for advisory board work and lectures, and non-financial (travel) support from Actelion, grants, personal fees for consultancy and lectures, and non-financial (travel) support from Boehringer Ingelheim and Roche, personal fees for advisory board and data monitoring committee work from Bayer/MSD and Galapagos, personal fees for adjudication committee work from Gilead, personal fees for advisory board work and lectures from Novartis, personal fees for lectures from Sanofi, personal fees for data monitoring and steering committee work from Promedior, personal fees for data monitoring committee work from Celgene and Galecto, outside the submitted work. E. Bendstrup reports grants and personal fees from Boehringer Ingelheim and Roche, outside the submitted work.

\section{References}

1 Ley B, Collard HR, King TE Jr. Clinical course and prediction of survival in idiopathic pulmonary fibrosis. Am J Respir Crit Care Med 2011; 183: 431-440.

2 Kreuter M, Koegler $\mathrm{H}$, Trampisch $\mathrm{M}$, et al. Differing severities of acute exacerbations of idiopathic pulmonary fibrosis (IPF): insights from the INPULSIS(R) trials. Respir Res 2019; 20: 71.

3 Collard HR, Ryerson CJ, Corte TJ, et al. Acute Exacerbation of Idiopathic Pulmonary Fibrosis. An International Working Group Report. Am J Respir Crit Care Med 2016; 194: 265-275.

4 Collard HR, Richeldi L, Kim DS, et al. Acute exacerbations in the INPULSIS trials of nintedanib in idiopathic pulmonary fibrosis. Eur Respir J 2017; 49: 1601339.

5 Ley B, Swigris J, Day BM, et al. Pirfenidone reduces respiratory-related hospitalizations in idiopathic pulmonary fibrosis. Am J Respir Crit Care Med 2017; 196: 756-761.

6 Raghu G, Collard HR, Egan JJ, et al. An official ATS/ERS/JRS/ALAT statement: idiopathic pulmonary fibrosis: evidence-based guidelines for diagnosis and management. Am J Respir Crit Care Med 2011; 183: 788-824.

7 Walsh SLF, Maher TM, Kolb M, et al. Diagnostic accuracy of a clinical diagnosis of idiopathic pulmonary fibrosis: an international case-cohort study. Eur Respir J 2017; 50: 1700936.

8 Akira M, Kozuka T, Yamamoto S, et al. Computed tomography findings in acute exacerbation of idiopathic pulmonary fibrosis. Am J Respir Crit Care Med 2008; 178: 372-378.

9 Faverio P, De Giacomi F, Sardella L, et al. Management of acute respiratory failure in interstitial lung diseases: overview and clinical insights. BMC Pulm Med 2018; 18: 70.

10 Navaratnam V, Fogarty AW, McKeever T, et al. Presence of a prothrombotic state in people with idiopathic pulmonary fibrosis: a population-based case-control study. Thorax 2014; 69: 207-215.

11 Ohshimo S, Ishikawa N, Horimasu Y, et al. Baseline KL-6 predicts increased risk for acute exacerbation of idiopathic pulmonary fibrosis. Respir Med 2014; 108: 1031-1039.

12 Nikaido T, Tanino Y, Wang X, et al. Serum decorin is a potential prognostic biomarker in patients with acute exacerbation of idiopathic pulmonary fibrosis. J Thorac Dis 2018; 10: 5346-5358.

13 Molyneaux PL, Cox MJ, Wells AU, et al. Changes in the respiratory microbiome during acute exacerbations of idiopathic pulmonary fibrosis. Respir Res 2017; 18: 29.

14 Kawamura K, Ichikado K, Yasuda Y, et al. Azithromycin for idiopathic acute exacerbation of idiopathic pulmonary fibrosis: a retrospective single-center study. BMC Pulm Med 2017; 17: 94.

15 Ding J, Chen Z, Feng K. Procalcitonin-guided antibiotic use in acute exacerbations of idiopathic pulmonary fibrosis. Int J Med Sci 2013; 10: 903-907.

16 Wedzicha JA. Role of viruses in exacerbations of chronic obstructive pulmonary disease. Proc Am Thorac Soc 2004; 1: 115-120. 
17 Wootton SC, Kim DS, Kondoh Y, et al. Viral infection in acute exacerbation of idiopathic pulmonary fibrosis. Am J Respir Crit Care Med 2011; 183: 1698-1702.

18 Saraya T, Kimura H, Kurai D, et al. Clinical significance of respiratory virus detection in patients with acute exacerbation of interstitial lung diseases. Respir Med 2018; 136: 88-92.

19 Idiopathic Pulmonary Fibrosis Clinical Research Network, Raghu G, Anstrom KJ, et al. Prednisone, azathioprine, and N-acetylcysteine for pulmonary fibrosis. N Engl J Med 2012; 366: 1968-1977.

20 Papiris SA, Kagouridis K, Kolilekas L, et al. Survival in Idiopathic pulmonary fibrosis acute exacerbations: the non-steroid approach. BMC Pulm Med 2015; 15: 162.

21 Sakamoto S, Homma S, Miyamoto A, et al. Cyclosporin A in the treatment of acute exacerbation of idiopathic pulmonary fibrosis. Intern Med 2010; 49: 109-115.

22 Novelli L, Ruggiero R, De Giacomi F, et al. Corticosteroid and cyclophosphamide in acute exacerbation of idiopathic pulmonary fibrosis: a single center experience and literature review. Sarcoidosis Vasc Diffuse Lung Dis 2016; 33: 385-391.

23 Horita N, Akahane M, Okada Y, et al. Tacrolimus and steroid treatment for acute exacerbation of idiopathic pulmonary fibrosis. Intern Med 2011; 50: 189-195.

24 Donahoe M, Valentine VG, Chien N, et al. Autoantibody-targeted treatments for acute exacerbations of idiopathic pulmonary fibrosis. PLoS One 2015; 10: e0127771.

25 Kolb M, Kirschner J, Riedel W, et al. Cyclophosphamide pulse therapy in idiopathic pulmonary fibrosis. Eur Respir J 1998; 12: 1409-1414.

26 Richeldi L, du Bois RM, Raghu G, et al. Efficacy and safety of nintedanib in idiopathic pulmonary fibrosis. $N$ Engl J Med 2014; 370: 2071-2082.

27 Cottin V, Annesi-Maesano I, Gunther A, et al. The Ariane-IPF ERS Clinical Research Collaboration: seeking collaboration through launch of a federation of European registries on idiopathic pulmonary fibrosis. Eur Respir J 2019; 53: 1900539 .

28 Arcadu A, Moua T. Bronchoscopy assessment of acute respiratory failure in interstitial lung disease. Respirology 2017; 22: 352-359.

29 Teramachi R, Kondoh Y, Kataoka K, et al. Outcomes with newly proposed classification of acute respiratory deterioration in idiopathic pulmonary fibrosis. Respir Med 2018; 143: 147-152.

30 Rangappa P, Moran JL. Outcomes of patients admitted to the intensive care unit with idiopathic pulmonary fibrosis. Crit Care Resusc 2009; 11: 102-109.

31 Raghu G, Rochwerg B, Zhang Y, et al. An Official ATS/ERS/JRS/ALAT Clinical Practice Guideline: Treatment of Idiopathic Pulmonary Fibrosis. An Update of the 2011 Clinical Practice Guideline. Am J Respir Crit Care Med 2015; 192: e3-19.

32 Yokoyama $\mathrm{T}$, Kondoh $\mathrm{Y}$, Taniguchi $\mathrm{H}$, et al. Noninvasive ventilation in acute exacerbation of idiopathic pulmonary fibrosis. Intern Med 2010; 49: 1509-1514.

33 Trudzinski FC, Kaestner F, Schafers HJ, et al. Outcome of patients with interstitial lung disease treated with extracorporeal membrane oxygenation for acute respiratory failure. Am J Respir Crit Care Med 2016; 193: $527-533$.

34 Mooney JJ, Raimundo K, Chang E, et al. Mechanical ventilation in idiopathic pulmonary fibrosis: a nationwide analysis of ventilator use, outcomes, and resource burden. BMC Pulm Med 2017; 17: 84.

35 Cottin V, Crestani B, Valeyre D, et al. Diagnosis and management of idiopathic pulmonary fibrosis: French practical guidelines. Eur Respir Rev 2014; 23: 193-214.

36 Lee JS, Song JW, Wolters PJ, et al. Bronchoalveolar lavage pepsin in acute exacerbation of idiopathic pulmonary fibrosis. Eur Respir J 2012; 39: 352-358.

37 Lee JS, Ryu JH, Elicker BM, et al. Gastroesophageal reflux therapy is associated with longer survival in patients with idiopathic pulmonary fibrosis. Am J Respir Crit Care Med 2011; 184: 1390-1394.

38 Lee JS, Collard HR, Anstrom KJ, et al. Anti-acid treatment and disease progression in idiopathic pulmonary fibrosis: an analysis of data from three randomised controlled trials. Lancet Respir Med 2013; 1: 369-376.

39 Kreuter M, Wuyts W, Renzoni E, et al. Antacid therapy and disease outcomes in idiopathic pulmonary fibrosis: a pooled analysis. Lancet Respir Med 2016; 4: 381-389.

40 Costabel U, Behr J, Crestani B, et al. Anti-acid therapy in idiopathic pulmonary fibrosis: insights from the INPULSIS(R) trials. Respir Res 2018; 19: 167.

41 Cottin V LH, Luppi F, Le Maulf F, et al. Effect of baseline corticosteroid medication on reduction in FVC decline with nintedanib. Eur Respir J 2015; 46: Suppl. 59, OA4498.

42 Noth I, Anstrom KJ, Calvert SB, et al. A placebo-controlled randomized trial of warfarin in idiopathic pulmonary fibrosis. Am J Respir Crit Care Med 2012; 186: 88-95.

43 Kreuter M, Wijsenbeek MS, Vasakova M, et al. Unfavourable effects of medically indicated oral anticoagulants on survival in idiopathic pulmonary fibrosis: methodological concerns. Eur Respir J 2016; 48: 1524-1526. 